\title{
CSPs with Global Modular Constraints: Algorithms and Hardness via Polynomial Representations*
}

\author{
Joshua Brakensiek ${ }^{\dagger}$ \\ Stanford University \\ Stanford, USA \\ jbrakens@stanford.edu
}

\author{
Sivakanth Gopi ${ }^{\ddagger}$ \\ Microsoft Research \\ Redmond, USA \\ sigopi@microsoft.com
}

\author{
Venkatesan Guruswami $§$ \\ Carnegie Mellon University \\ Pittsburgh, USA \\ venkatg@cs.cmu.edu
}

\begin{abstract}
We study the complexity of Boolean constraint satisfaction problems (CSPs) when the assignment must have Hamming weight in some congruence class modulo $M$, for various choices of the modulus $M$. Due to the known classification of tractable Boolean CSPs, this mainly reduces to the study of three cases: 2-SAT, HORN-SAT, and LIN-2 (linear equations mod 2). We classify the moduli $M$ for which these respective problems are polynomial time solvable, and when they are not (assuming the ETH). Our study reveals that this modular constraint lends a surprising richness to these classic, well-studied problems, with interesting broader connections to complexity theory and coding theory. The HORN-SAT case is connected to the covering complexity of polynomials representing the NAND function $\bmod M$. The LIN-2 case is tied to the sparsity of polynomials representing the OR function $\bmod M$, which in turn has connections to modular weight distribution properties of linear codes and locally decodable codes. In both cases, the analysis of our algorithm as well as the hardness reduction rely on these polynomial representations, highlighting an interesting algebraic common ground between hard cases for our algorithms and the gadgets which show hardness. These new complexity measures of polynomial representations merit further study.

The inspiration for our study comes from a recent work by Nägele, Sudakov, and Zenklusen on submodular minimization with a global congruence constraint. Our algorithm for HORN-SAT has strong similarities to their algorithm, and in particular identical kind of set systems arise in both cases. Our connection to polynomial representations leads to a simpler analysis of such set systems, and also sheds light on (but does not resolve) the complexity of submodular minimization with a congruency requirement modulo a composite $M$.
\end{abstract}

\footnotetext{
${ }^{*}$ Full version of this paper is available at https://arxiv.org/abs/1902.04740.

$\dagger$ Portions of this work were done while at Carnegie Mellon University and during a visit to Microsoft Research, Redmond. Research supported in part by NSF CCF-1526092 and an NSF Graduate Research Fellowship.

¥Part of the research was done while the author was a student at Princeton University where he was supported by NSF CAREER award 1451191 and NSF grant CCF-1523816. ${ }^{\S}$ Research supported in part by NSF grants CCF-1422045 and CCF-1526092.

Permission to make digital or hard copies of all or part of this work for personal or classroom use is granted without fee provided that copies are not made or distributed for profit or commercial advantage and that copies bear this notice and the full citation on the first page. Copyrights for components of this work owned by others than the author(s) must be honored. Abstracting with credit is permitted. To copy otherwise, or republish, to post on servers or to redistribute to lists, requires prior specific permission and/or a fee. Request permissions from permissions@acm.org.

STOC '19, June 23-26, 2019, Phoenix, AZ, USA

(C) 2019 Copyright held by the owner/author(s). Publication rights licensed to ACM. ACM ISBN 978-1-4503-6705-9/19/06 . .\$15.00

https://doi.org/10.1145/3313276.3316401
}

\section{CCS CONCEPTS}

- Theory of computation $\rightarrow$ Computational complexity and cryptography; Problems, reductions and completeness; Design and analysis of algorithms; Error-correcting codes; Complexity theory and logic.

\section{KEYWORDS}

Constraint satisfaction problems, Modular constraints, Polynomial representations, Exponential time hypothesis (ETH), Submodular minimization, Matching vector families

\section{ACM Reference Format:}

Joshua Brakensiek, Sivakanth Gopi, and Venkatesan Guruswami. 2019. CSPs with Global Modular Constraints: Algorithms and Hardness via Polynomial Representations. In Proceedings of the 51st Annual ACM SIGACT Symposium on the Theory of Computing (STOC '19), June 23-26, 2019, Phoenix, AZ, USA. ACM, New York, NY, USA, 12 pages. https://doi.org/10.1145/3313276.3316401

\section{INTRODUCTION}

We study how the complexity of tractable cases of Boolean constraint satisfaction problems, namely 2-SAT, HORN-SAT and LIN-2 (linear equations over $\mathbb{F}_{2}$ ), is affected when we seek a solution that obeys a global modular constraint, such as Hamming weight being divisible by some modulus $M$. As our work reveals, this seemingly simple twist lends a remarkable amount of richness to these classic problems, raising new questions concerning polynomial representations of simple Boolean functions that form the common meeting ground of both algorithmic and hardness results.

The inspiration for our study comes from a beautiful recent work on minimizing a submodular function in the presence of a global modular constraint [25]. This framework captures questions such as: Given a graph $G$, find the minimum cut one of whose sides has size divisible by 6 . The complexity of this basic question remains open. Surprisingly, the same combinatorial set system that governed the complexity of the algorithms in [25] arises in our study of HORN-SAT with a modular constraint. We connect such set systems to polynomial representations of the NAND function, thereby shedding light on submodular minimization with a global constraint involving a composite modulus, a case not handled by [25]. We describe this connection, as well as the relation to integer programs of bounded modularity that partly motivated [25] in Section 1.3.4.

\subsection{CSPs and Modular CSPs}

We now describe some of our other, intrinsic motivations to study the modular variant of constraint satisfaction problems (CSPs). CSPs 
have a storied place in computational complexity, spurring several of its most influential developments including NP-completeness, Schaefer's dichotomy theorem [27], the PCP theorem and Khot's Unique Games conjecture (which together have led to the very rich field of inapproximability), and the algebraic program for studying CSPs inspired by the Feder-Vardi [16] and crystallized by Bulatov, Jeavons and Krokhin [5], which recently culminated in the resolution of the CSP dichotomy conjecture [7, 31].

One reason that CSPs receive so much attention is that the local nature of their constraints offers just the right amount of structure to aid the development of novel algorithmic and hardness techniques, which then often extend to more general settings. For instance, semidefinite programming which was first used in approximating the Max-Cut CSP, has been one of the most influential algorithmic tools in approximating a whole variety of problems. On the hardness side, the PCP theorem, which is a statement about hardness of approximating Max-CSP, in combination with clever gadgets has led to inapproximability results for covering, packing, cut, routing, and other classes of problems. Further, for the problem of satisfiability of CSPs, we have a precise understanding of the interplay between mathematical structure and tractability: efficient algorithms exist iff the problem admits non-trivial "polymorphisms" which are operations under which the solution space is closed.

One enhancement to the CSP framework would be to impose some global constraint on the solution. For example, one could impose a global cardinality constraint, such as an equal number of 0 's and 1's in the solution, or more generally a specified frequency for each value in the domain. This global condition is quite strict, often making many tractable CSPs NP-complete once these constraints are added. In fact, in the Boolean case the "hardest" problem that can be solved in polynomial time is (weighted)graph 2-coloring (by doing a simple dynamic program on the connected components) [12].

In the case of approximating a 2-SAT instance with a balanced cardinality constraint (an equal number of 0 s and 1s), it is NP-hard to solve [20]. In fact, the authors show that it is NP-hard to find an assignment satisfying a $\left(1-\varepsilon_{0}\right)$ fraction of clauses for some absolute constant $\varepsilon_{0}>0$. Further, this inapproximability holds for 2-SAT instances with Horn clauses, and thus also implies hardness of HORN-SAT with a balanced cardinality constraint.

This strictness of the constraints allowed for a full dichotomy (for any sized domain) to be proved long before the Feder-Vardi dichotomy was resolved, as most problems become NP-hard [8] Bulatov and Marx showed that the only tractable problems are those that are "non-crossing decomposable." Although the formal definition is a bit technical, informally such problems need to both be "convex" in that they are tractable in the second round of the Sherali-Adams hierarchy as well as "linear" in that they are solvable using a variant of Gaussian elimination (c.f., [3]). As those two types of algorithms typically solve quite different problems (think 2-SAT versus $3-\mathrm{XOR}$ ), the family of tractable cardinality CSPs is far less diverse than for "ordinary" CSPs.

The main focus of this work is to investigate CSPs with a much less strict global constraint, which we refer to as a modular (or congruency) constraint. We will restrict ourselves to the Boolean case in this work, and impose the requirement that the number of
Table 1: For each entry, guess whether the corresponding problem is solvable in polynomial time.

\begin{tabular}{r|r|r|r|r|r} 
Name & Constraint types & $M=3$ & $M=4$ & $M=6$ & $M=15$ \\
\hline 2-SAT-MOD \\
\hline HORN-SAT-MOD $_{M}$ & $x \vee y ; x=\neg y$ & & & & \\
\hline LIN-2-MOD \\
\hline & $x \oplus y \oplus z=0$ or 1 & & & & \\
\hline
\end{tabular}

1 's in the solution be congruent to $\ell$ modulo $M$, for some integers $\ell, M .{ }^{1}$ We refer to this class of problems as (Boolean) Mod-CSPs.

Informally, it is easy to see that any such Mod-CSP is at least as hard as the corresponding local CSP, as we can take a local CSP instance and add $M$ dummy variables not part of any clauses, so that the modular constraint is now trivially satisfiable. Conversely, these Mod-CSPs are at least as easy (up to polynomial factors) as the corresponding cardinality problem, because we can brute force the cardinality of $1 \mathrm{~s}$ by trying all $c_{1}=\ell \bmod M$.

By Schaefer's Boolean CSP dichotomy theorem [27], there are only three essentially different non-trivial tractable cases of Boolean CSP: 2-SAT, HORN-SAT, and LIN-2. We thus study each of these problems when we seek a solution of Hamming weight in some congruence class modulo $M$, for a fixed $M$. (When $M$ can grow with the input, these problems become NP-hard as one can encode a global cardinality constraint [12].) Our goal is to classify the cases which are polynomial time solvable, as a function of $M$. In order to better appreciate the difficulty of this endeavor, we encourage the reader to not peek ahead and write down a guess for each of the cases listed in Table 1. (Note that 2-SAT-MOD $M$ refers to 2-SAT with the global constraint modulo $M$, etc.)

Our work hinges on several connections which makes our investigation interesting beyond the specific application to modular CSPs. The complexity of the problems (except 2-SAT-MOD $M$ ) are tied to the parameters of certain polynomial representations - lower bounds on such representations yields our algorithmic guarantees, and at the same the existence of efficient representations implies hardness results. Thus the work illustrates an interesting duality between algorithms and hardness as originating from the same object. The relevant complexity measures for polynomial representations are novel and deserve further study. As the particular choice of complexity measure for each problem seems closely linked to the underlying polymorphisms of the CSP, we hope that initiating such a study could help bring together computational complexity theorists and specialists in the algebraic theory of CSPs.

The result for linear equations has interesting connections to coding theory, namely the extremal dimension of binary linear codes whose codewords have modular restrictions on their weights (which relates to concepts like doubly even codes that have been studied in coding theory), as well as to locally decodable codes, via relationship between polynomial representations and matching vector families and the Polynomial Freiman-Ruzsa (PFR) conjecture in additive combinatorics.

${ }^{1}$ Our actual setup is a bit more general, associating a weight from an Abelian group for
each variable-value pair, and requiring that the sum of the weights equals some value. 
Table 2: Summary of main results

\begin{tabular}{|c|c|c|c|c|}
\hline & \multicolumn{2}{|c|}{$M$ is a prime power } & \multicolumn{2}{c|}{$M$ has $r$ distinct prime factors $(r \geqslant 2)$} \\
\cline { 2 - 5 } & Algorithm & Hardness & Algorithm & Hardness \\
\hline 2-SAT-MOD \\
\hline & $n^{M+O(1)}$ & - & $n^{M+O(1)}$ & - \\
\hline HORN-SAT-MOD $_{M}(n)$ & $n^{M+O(1)}$ & $n^{\Omega\left(\frac{M}{\log M}\right)}$ & - & $\exp \left(\Omega_{M}\left(\left(\frac{\log n}{\log \log n}\right)^{r}\right)\right)$ \\
\hline LIN-2-MOD $_{M}(n)^{4}$ & $n^{M+O(1)}$ & $n^{\Omega\left(\frac{M}{\log M}\right)}$ & $\exp \left(O_{M}\left(\frac{n}{\log n}\right)\right)$ & $\exp \left(\Omega_{M}\left(\left(\frac{\log n}{\log \log n}\right)^{r}\right)\right)$ \\
\hline
\end{tabular}

\subsection{Our Results}

For 2-SAT-MOD ${ }_{M}$, HORN-SAT-MOD $M$, and LIN-2-MOD $M$, we resolve the complexity, namely whether it belongs to or is unlikely to be polynomial time solvable, for all moduli. ${ }^{2}$ We will denote by 2-SAT-MOD $M(n)$ an instance of 2-SAT with $n$ variables and a global constraint modulo $M, \operatorname{HORN}-\mathrm{SAT}_{-} \mathrm{MOD}_{M}(n)$ and LIN-2-MOD $M(n)$ are similarly defined.

Theorem 1.1 (Informal STATEMENT of MAIn Results). Suppose we have a single global modular constraint with a fixed modulus $M$.

(1) 2-SAT-MOD $M(n)$ is polytime solvable for all moduli $M$.

(2) HORN-SAT-MOD $M(n)$ is polytime solvable when the modulus $M$ equals a prime power, and assuming ETH, cannot be solved in $n^{o\left((\log n / \log \log n)^{r-1}\right)}$ time when $M$ has $r$ distinct prime factors.

(3) $\mathrm{LIN}-2-\mathrm{MOD}_{M}(n)$ is randomized polytime solvable when the modulus $M=2^{\ell} p^{s}$ for some odd prime $p$ and $\ell \leqslant 2$, and assuming ETH, cannot be solved in $n^{o\left((\log n / \log \log n)^{r-1}\right)}$ when $M$ has $r$ distinct odd prime factors. Further, assuming the Polynomial Freiman-Ruzsa conjecture, there is an algorithm with runtime $\exp \left(O_{M}(n / \log n)\right)$ for all $M .^{3}$

The polynomial runtime grows like $n^{O(M)}$, and assuming ETH this cannot be improved to $n^{o(M / \log M)}$ for the case of HORN-SAT-MOD $M$ and LIN-2-MOD ${ }_{M}$. Moreover, for the LIN-2-MOD $M$ problem, the randomized polytime algorithms have quasipolynomial derandomizations.

For the one uncovered case of LIN-2-MOD $M(n)$, when $M=2^{\ell} p^{s}$ for an odd prime $p$ and $\ell>2$, we give a quasi-polynomial time algorithm with runtime $\exp \left(O_{M}\left((\log n)^{2^{\ell-1}-1}\right)\right)$. We are not sure if there should be a polynomial time algorithm also in this case.

Table 2 summarizes our results. We will assume $M \leqslant c n$ for some sufficiently small constant $c>0$. All the hardness results are assuming ETH. For simplicity, the results for LIN-2-MOD $M$ are only stated for odd $M$ and the running times are for randomized algorithms, the algorithm for general $M$ is assuming PFR conjecture.

Extensions to Multiple Modular Constraints. We also consider natural extensions of the three problems, where we allow a small number of $\bmod M$ constraints or the more general version where we allow $\ell$ constraints with different moduli $M_{1}, M_{2}, \ldots, M_{\ell}$. Our algorithm our 2-SAT-MOD $M$ is presented in this more general model

${ }^{2}$ Unfortunately, one uncovered case is LIN-2-MOD $M$ for moduli $M=2^{\ell} p^{s}$ for an odd prime $p$ and $\ell \geqslant 3$.

${ }^{3}$ We write $A=O_{M}(B)$ or $A \S_{M} B$ to say that $A \leqslant C_{M} B$ for some constant $C_{M}>0$ which depends only on $M$ and not on any other parameters. $A=\Omega_{M}(B), A \gtrsim_{M}$ $B, A=o_{M}(B), A=\omega_{M}(B)$ are similarly defined.

${ }^{4}$ For LIN-2-MOD $M$, we are only stating the results for odd $M$ and the algorithms are randomized. The running time of the algorithm for non-prime-power $M$ is conditioned on Polynomial Freiman-Ruzsa conjecture.
For HORN-SAT and LIN-2, we show that these general versions can be reduced to the basic version with a single $\bmod M$ constraint, without increasing the size of the instance too much. For example we can reduce an instance of HORN-SAT over $n$ variables with $\ell$ constraints modulo $M$ to an instance of HORN-SAT-MOD $M$ over $n^{O_{M}(\ell)}$ variables. Note that once $\ell$ becomes linear in $n$, these problems are NP-hard, so an exponential dependence in $\ell$ is necessary. A similar statement also holds for LIN-2 with multiple modular constraints.

Completing a Classification of Boolean Mod-CSPs. Although HORN-SAT, LIN-2, and 2-SAT are the most important CSPs that need to be analyzed, some additional care is needed to extend these classifications to all Boolean CSPs. This work is done in Appendix A of the full version, where we classify the computational complexity of all Boolean Mod-CSPs. The main observation is to use a classification by Post [26] of the polymorphisms of Boolean CSPs. Using this classification, we can show that when $M$ is a prime power, the Mod-CSP problem is always either in RP or is NP-complete. On the other hand, when $M$ is divisible by distinct primes, the classification becomes a bit more difficult. The main difficulty is that we have an upper bound for 2-SAT-MOD $M$, but lower bounds on HORN-SAT-MOD $M$ and LIN-2-MOD $M$ (when $M$ is divisible by distinct odd primes). Because of the confluence of lower and upper bounds, there are some additional tractable cases that show up, which are similar in structure to 2-SAT. See Section A.4 of the full version for more details.

\subsection{Our Techniques and Connections}

We now give brief overviews of our approach to establish Theorem 1.1. We discuss each of the three constraint types in turn.

1.3.1 2-SAT-MOD ${ }_{M}$ and Recursive Methods. For 2-SAT-MOD ${ }_{M}$, our algorithm is recursive. The one key idea is that we work with a more general form of modular constraint to make the recursion work, one that allows the Hamming weight to belong to a subset $S$ of congruence classes (rather than a single value). Standard methods, with some care to update the modular constraint, allow us to reduce to the case when the "implication graph" on the literals of the 2-SAT instance is a DAG. We then select a literal $y$ with no outgoing edge, and first set it to 1 (which doesn't impact any other literal), and solve the 2-SAT instance on the remaining variables with an updated modular constraint (that takes into account the setting of $y$ ). If this succeeds, we can output this assignment and be done.

Otherwise we set $y=0$, which forces all literals which have a path to $y$ in the DAG also to 0 . We can update the modular constraint accordingly, but note that in the end we are allowed to flip $y$ to 1 and the 2-SAT instance will still be satisfied. While there is no need to do this for normal 2-SAT, this flip might allow us to satisfy the modular constraint. As a result, we allow the set $S$ of congruence classes in the recursive call to also include this possibility. This is the reason why we need to work with the more general form of modular constraint. To implement this idea to run in polynomial time is a bit subtle, as naively we could reduce an instance with $n$ variables to two instances with $n-O(1)$ variables leading to exponential runtime. To avoid this pitfall, we track the size of the allowed moduli $S$, and argue that if it doesn't increase in the second recursive call (one where we set $y=0$ ), we can truncate that call 
and return no solution for that branch. This is justified because any valid solution with $y=0$ remains valid when $y=1$, and the former is already ruled out in the first call. The increase in $|S|$ in one of the recursive calls implies a polynomially bounded solution to the recurrence for the runtime, with exponent at most $M-1$.

1.3.2 HORN-SAT-MOD $M$ and Covering Number of NAND $\bmod M$ over $\{0,1\}$ Basis. For HORN-SAT-MOD $M$, i.e., HORN-SAT with a single global linear constraint modulo $M$, our algorithm and analysis is very different from the 2-SAT case. An important property of the HORN-SAT instance is that the set of solutions in intersectionclosed. Given an instance $\Psi$ of HORN-SAT and a subset $S$ of its variables, one can efficiently find the minimal solution among all solutions of $\Psi$ which set the variables of $S$ to 1 , this is called the FindMinimal $(\Psi, S)$ routine. Now we run FindMinimal $(\Psi, S)$ on all subsets $S$ up to a give size $R$ and check if any of the outputs satisfy the modular constraint. If none of them satisfy the modular constraint, we claim that $\Psi$ has no solution which satisfies the modular constraint. The running time of this $R$-round algorithm is $n^{R+O(1)}$. If the $R$-round algorithm for HORN-SAT-MOD ${ }_{M}$ fails, then we show that it is because of a special kind of obstruction. To describe these obstructions, we will need a few definitions.

Definition 1.2. A multilinear polynomial $p\left(x_{1}, \ldots, x_{d}\right)$ is said to represent $\mathrm{NAND}_{d} \bmod M$ over $\{0,1\}^{d}$ if it has integer coefficients and

$$
p(x)\left\{\begin{array}{lll}
= & 0 & \bmod M \text { if } x=1 \\
\neq & 0 & \bmod M \text { if } x \in\{0,1\}^{d} \backslash\{1\}
\end{array}\right.
$$

where $\mathbf{1}$ is the all ones vector.

Definition 1.3 (Covering number). The covering number of a multilinear polynomial $p(x)$, denoted by $\operatorname{cov}(p)$, is the minimum number of monomials of $p$ one can choose such that every variable that appears in $p$ appears in one of them.

Note that the covering number is the minimum set cover of the family of subsets of variables given by the monomials. An obstruction for the $R$-round algorithm to solve HORN-SAT-MOD $M(n)$ correctly is a polynomial $p$ which represents NAND $\bmod M$ with at most $n+1$ monomials and $\operatorname{cov}(p)>R$. Therefore we have the following proposition.

PROPOSITION 1.4. If every polynomial $p$ which represents NAND $\bmod M$ over $\{0,1\}$ basis with $n+1$ monomials has $\operatorname{cov}(p) \leqslant R(n)$, then there exists an $n^{R+O(1)}$-time algorithm for HORN-SAT-MOD $M(n)$.

When $M$ is a Prime Power. In this case, we can show that any polynomial which represents NAND $\bmod M$ should have covering number at most $M-1$. Note that this bound is independent of the number of monomials in the polynomial. This implies that our algorithm with $R=M-1$ rounds solves HORN-SAT-MOD $M(n)$ correctly.

When $M$ has $r \geqslant 2$ Distinct Prime Factors. In this case, it turns out that there are obstructions for any constant round algorithm. More precisely, there are polynomials which represent NAND modulo $M$ with $n$ monomials, but their covering number is at least $\Omega\left((\log n / \log \log n)^{r-1}\right)$. Such polynomials can be obtained from polynomials of degree $O\left(d^{1 / r}\right)$ which represent NAND ${ }_{d} \bmod M$ constructed by Barrington, Beigel and Rudich [2]. This implies that the $R$-round algorithm will not work for the choice of $R=$ $o\left((\log n / \log \log n)^{r-1}\right)$. This by itself does not show hardness of HORN-SAT-MOD $M$, it just shows that our algorithm doesn't work with constant rounds. But it turns out that we can use low-degree polynomial representations of NAND $\bmod M$ directly as a gadget to reduce 3-SAT to HORN-SAT-MOD $M$ without blowing up the size too much. More precisely, we show the following hardness result.

Proposition 1.5. Suppose there exists a polynomial which represents $\mathrm{NAND}_{d} \bmod M$ over $\{0,1\}^{d}$ with degree $\triangle$. Assuming ETH, HORN-SAT-MOD $M(n)$ requires $2^{\Omega(d)}$ time for somen $=(e d / \Delta)^{O(\Delta)}$.

Using that $\Delta=O\left(d^{1 / r}\right)$ from [2], we get an ETH-hardness of $\exp \left(\Omega_{M}\left((\log n / \log \log n)^{r}\right)\right)$ time for HORN-SAT-MOD $M(n)$. Better upper bounds on $\Delta$ lead to better hardness results. The best known lower bound on $\Delta$ is $\Omega_{M}\left((\log d)^{1 /(r-1)}\right)$ due to Barrington and Tardos [28]. If there is a polynomial whose degree matches this lower bound, then assuming ETH, we can get an even better $\exp \left(\exp \left((\log n)^{1-1 / r}\right)\right)$ hardness for HORN-SAT-MOD $M(n)$.

What about sub-exponential time algorithms? We conjecture that the covering number of any polynomial which represents NAND $\bmod M$ with $n$ monomials should be $n^{o_{M}(1)}$ for any fixed $M$. If true, this would imply an $\exp \left(n^{o_{M}(1)}\right)$-time algorithm for HORN-SAT-MOD $M(n)$. We give evidence towards our conjecture by showing that the fractional covering number (which is an LP relaxation of covering number) of any such polynomial is indeed $n^{o_{M}(1)}$.

1.3.3 LIN-2-MOD $M$ and Sparsity of the OR $\bmod M$ over $\{-1,1\}$ Basis. The algorithm and analysis for LIN-2-MOD $M$ has strong similarities to the analysis for HORN-SAT-MOD $M$. The algorithm is quite simple to state: sample $T$ uniformly random solutions to the linear system over $\mathbb{F}_{2}$ and check if any of them satisfy the modular constraint.

To analyze how many samples $T$ to check so that the algorithm is correct with high probability, we need to prove that for any $d$-dimensional affine subspace of $\mathbb{F}_{2}^{n}$, there are either 0 points in this space satisfying the modular constraint or there are at least $2^{d} / f(n, M)$ such points. Then $T=O(f(n, M))$ samples would suffice. By a simple reduction, it suffices to bound the maximal dimension $\mathcal{D}(n, M)$ such that a $\mathcal{D}$-dimensional affine subspace of $\mathbb{F}_{2}^{n}$ has exactly one element whose Hamming weight is $a \bmod M$ for some a. Quantitatively, we show that $f(n, M) \leqslant O\left(2^{\mathcal{D}(n, m)}\right)$. In other words, the obstructions for our algorithm are large affine subspaces which have exactly one point which satisfies a linear constraint modulo $M$. When $M$ is a power of 2 , we prove that $\mathcal{D}(n, M) \leqslant M-1$. For $M=2^{\ell} M^{\prime}$ for some odd $M^{\prime} \geqslant 3$, we can get upper bounds on $\mathcal{D}(n, M)$ from upper bounds on $\mathcal{D}\left(n, M^{\prime}\right)$. So we can only focus on odd $M$. The obstructions for odd $M$ can be represented using polynomials like we did in the HORN-SAT-MOD $M$ case.

Definition 1.6. A polynomial $p\left(x_{1}, \ldots, x_{d}\right)$ is said to represent $\mathrm{OR}_{d} \bmod M$ over $\{-1,1\}^{d}$ if it has integer coefficients and

$$
p(x)\left\{\begin{array}{lll}
= & 0 & \bmod M \text { if } x=1 \\
\neq & 0 & \bmod M \text { if } x \in\{-1,1\}^{d} \backslash\{1\}
\end{array}\right.
$$

where $\mathbf{1}$ is the all ones vector. 
The existence of a $d$-dimensional affine subspace of $\mathbb{F}_{2}^{n}$ with exactly one point satisfying the $\bmod M$ constraint is equivalent to an $(n+1)$-sparse polynomial representation of $\mathrm{OR}_{d} \bmod M$ over $\{-1,1\}^{d}$. Thus, lower bounds on the sparsity of representations of OR $\bmod M$ in the $\{-1,1\}$ basis imply upper bounds on $\mathcal{D}(n, M)$ and the runtime of our randomized algorithm. More precisely, we have the following proposition.

Proposition 1.7. Let $M$ be odd, if there is no polynomial which represents $\mathrm{OR}_{d} \bmod M$ over $\{-1,1\}^{d}$ with at most $n+1$ monomials, then there exists a $O\left(2^{d}{ }_{n}(1)\right)$ time randomized algorithm for LIN-2-MOD $M(n)$.

Connections to circuit complexity and LDCs. Note that an $s$ sparse polynomial which represents $\mathrm{OR}_{d} \bmod M$ over $\{-1,1\}^{d}$ is equivalent to a two layer $\mathrm{MOD}_{M} \circ \mathrm{MOD}_{2}$ circuit which computes OR over $\{0,1\}^{d}$ with $s M_{2}$-gates at the bottom level connected to a $\mathrm{MOD}_{M}$ gate at the top. ${ }^{5}$ Circuits made up of such MOD gates are extensively studied in circuit lower bounds $[9,10]$. An $s$-sparse polynomial representation of $\mathrm{OR}_{d} \bmod M$ over $\{-1,1\}^{d}$ can be used to construct a matching vector family (MVF) of size $2^{d}$ over $(\mathbb{Z} / M \mathbb{Z})^{s}$. MVFs of large size and small dimension can be used to construct good locally decodable codes (LDCs) and private information retrieval (PIR) schemes [13-15, 30]. So lower bounds for LDCs or upper bounds on the size of MVFs imply lower bounds on the sparsity of polynomials which represent $\mathrm{OR}_{d} \bmod M$ over $\{-1,1\}^{d}$. Concretely, superpolynomial lower bounds for the length of constant-query LDCs would imply $2^{o(n)}$ time algorithms for LIN-2-MOD $M(n)$ when $M$ is any constant; we find this connection quite surprising!

When $M$ is an odd prime power. In this case, we can show that the sparsity of a polynomial which represents $\mathrm{OR}_{d} \bmod M$ over $\{-1,1\}^{d}$ is at least $2^{d /(M-1)}$, which is nearly tight. In other words, $\mathcal{D}(n, M) \leqslant(M-1) \log n$. This immediately implies a $n^{M+O(1)}$-time randomized algorithm for LIN-2-MOD $M$.

When $M$ has $r \geqslant 2$ odd prime factors. The results of [2] imply that there exist degree $O\left(d^{1 / r}\right)$ polynomials which represent $\mathrm{OR}_{d}$ $\bmod M$ over $\{-1,1\}^{d}$, therefore these polynomials have at most $n=\exp \left(O\left(d^{1 / r} \log d\right)\right)$ monomials. In other words, $\mathcal{D}(n, M) \gtrsim$ $(\log n / \log \log n)^{r}$. Thus there are obstructions for our algorithm to run in polynomial time. This by itself does not show hardness. But, similar to the HORN-SAT-MOD $M$ case, we can directly use lowdegree polynomials which represent $\mathrm{OR}_{d} \bmod M$ over $\{-1,1\}^{d}$ to reduce 3-SAT to LIN-2-MOD $M$ without increasing the size too much.

Proposition 1.8. Suppose there exists a polynomial of degree $\Delta$ which represents $\mathrm{OR}_{d} \bmod M$ over $\{-1,1\}^{d}$. Assuming ETH, LIN-2-MOD $M(n)$ takes $2^{\Omega(d)}$ time for some $n=(\text { ed } / \Delta)^{O(\Delta)}$.

Using that $\Delta=O\left(d^{1 / r}\right)$ from [2], we get an ETH-hardness of $\exp \left(\Omega_{M}\left((\log n / \log \log n)^{r}\right)\right)$ time for LIN-2-MOD $M(n)$. Better upper bounds on $\Delta$ lead to better hardness results. The best known lower bound on $\Delta$ is $\Omega_{M}\left((\log d)^{1 /(r-1)}\right)$ due to Barrington and Tardos [28]. If there is a polynomial whose degree matches this lower

${ }^{5}$ Here a $M \mathrm{MD}_{M}$ gate outputs 0 if the number of 1 -inputs is a multiple of $M$ and outputs 1 otherwise. bound, then assuming ETH, we can get $\exp \left(\exp \left((\log n)^{1-1 / r}\right)\right)$ time hardness for LIN-2-MOD $M(n)$.

What about subexponential time algorithms? Unfortunately, we do not know any unconditional superlinear (i.e. $\omega(d))$ lower bounds on the sparsity of polynomials which represent $\mathrm{OR}_{d} \bmod M$ over $\{-1,1\}^{d}$. Such a lower bound would imply $2^{o(n)}$-time algorithms for LIN-2-MOD $M(n)$ for any constant $M$. But using the connection to MVFs, and an upper bound on the size of MVFS due to [4] assuming Polynomial Freiman-Ruzsa (PFR) conjecture from additive combinatorics, we can conclude that the sparsity of a polynomial representing $\mathrm{OR}_{d} \bmod M$ over $\{-1,1\}^{d}$ is at least $\Omega_{M}(d \log d)$ under the same conjecture. This implies an $\exp \left(O_{M}(n / \log n)\right)$-time algorithm for LIN-2-MOD $M(n)$ assuming the PFR conjecture.

1.3.4 Connections to Submodular Minimization and Integer Programming. A classic result in combinatorial optimization is that integer linear programs which have a totally unimodular constraint matrix, i.e., every square submatrix has determinant in $\{-1,0,1\}$, can be solved in polynomial time. This is because the vertices of the feasible polytope of such a constraint matrix are integral. Recently this result has been extended to totally bimodular constraint matrices, where every square submatrix has determinant in $\{-2,-1,0,1,2\}[1]$. Such results have inspired a conjecture that any integer program for which every square submatrix has determinant bounded in absolute value by $M$, can be solved in $n^{O_{M}(1)}$ time.

A recent paper by Nägele, Sudakov and Zenklusen [25] tries to lay the groundwork for proving this conjecture by considering a special case. This special case is finding the minimum cut in a directed graph such that the number of vertices on one side of the cut satisfies a modular condition modulo $M$. As cut functions of directed graphs are submodular, they generalized this question to the following algorithmic problem, denoted by SUBMOD-MIN-MOD $M(n)$ : Given $a, M$, minimize a submodular function $f:\{0,1\}^{n} \rightarrow \mathbb{Z}$ (given oracle access) over all $x$ such that $\sum_{i=1}^{n} x_{i}=a \bmod M .^{6}$

Our algorithm for HORN-SAT-MOD $M$ is actually inspired by the algorithm from [25] for SUBMOD-MIN-MOD $M(n)$. They show that the only obstructions to their $R$-round algorithm to work correctly are certain set families they called $(M, R, d)$-systems. They then showed that when $M$ is a prime power, $(M, M-1, d)$-systems do not exist for any $d$. This implies an $n^{O(M)}$-time algorithm for SUBMOD-MIN-MOD $M$ when $M$ is a prime power. They asked if a polynomial time algorithm exists for SUBMOD-MIN-MOD $M$ when $M$ has multiple prime factors.

We observe that $(M, R, d)$-systems are equivalent to polynomial representations of NAND $\bmod M$ over $\{0,1\}$ basis with $d$ monomials and covering number greater than $R$. Thus the obstructions for their algorithm for SUBMOD-MIN-MOD $M$ and our algorithm for HORN-SAT-MOD $M$ are exactly the same! Thus we can give a simpler proof of their result for prime power $M$, and also throw new light on SUBMOD-MIN-MOD $M$ when $M$ has multiple prime factors. In particular, if $M$ has $r$ distinct prime factors, this implies that there are obstructions for their $R$-round algorithm to work for SUBMOD-MIN-MOD $M(n)$ for $R=o_{M}\left((\log n / \log \log n)^{r-1}\right)$. This answers an open question from their paper and explains why

${ }^{6}$ A function $f:\{0,1\}^{n} \rightarrow \mathbb{Z}$ is called submodular if for all $x, y \in\{0,1\}^{n}, f(x)+$ $f(y) \geqslant f(x \vee y)+f(x \wedge y)$, where $\vee$ and $\wedge$ are bitwise OR and AND. 
they couldn't extend their algorithm for any constant $M$. If true, our conjecture that the covering number of a polynomial which represents NAND $\bmod M$ with $n$ monomials is $n^{o_{M}(1)}$, implies an $\exp \left(n^{o_{M}(1)}\right)$ time algorithm for SUBMOD-MIN-MOD $M$ for any $M$. We also make a conjecture about the existence of certain submodular functions, which would allow us to prove superpolynomial ETH-hardness results for SUBMOD-MIN-MOD $M$ when $M$ has multiple prime factors. It would be interesting if our methods have any implications for hardness of solving integer linear programs with bounded-minor constraint matrices, our results suggest that something different can happen at $M=6$.

\subsection{Future Directions}

Given that our work is the first to study the effect of global modular constraints on the tractability of CSPs, and our results unearth a rich picture rife with interesting connections to many central topics such as algebraic complexity measures of Boolean functions, coding theory, and combinatorial optimization, there are naturally many questions and directions for future work. We list a few below.

- Our work raises some intriguing new questions about polynomial representations modulo a composite number $M$. Can one prove an unconditional non-trivial (i.e, $\omega(d))$ lower bound on the sparsity of polynomial representing $\mathrm{OR}_{d} \bmod M$ over the $\{1,-1\}$-basis? Can one construct polynomial representations that are sparser than what is obtainable by simply appealing to the best known low-degree representations? For the covering complexity measure for polynomials representing NAND $\bmod M$ over the $\{0,1\}$ basis with $n$ monomials, can we prove an upper bound of $n^{o_{M}(1)}$ ? This would imply a sub-exponential time algorithm for

HORN-SAT-MOD $M$ and SUBMOD-MIN-MOD $M$ via our connections.

- The analysis of our algorithms for both $\mathrm{LIN}-2-\mathrm{MOD}_{M}$ and HORN-SAT-MOD $M$ use the sparsity and covering complexity of appropriate polynomial representations. On the other hand, our hardness results for these problems use the degree measure of these polynomials. Can one prove that sparsity and covering complexity directly dictate hardness as well? This would complete a very pleasing picture by giving matching algorithmic and hardness results and tying the complexity to some complexity parameter associated with polynomial representations.

- Can one show hardness of submodular minimization with a global mod 6 constraint? Constructing certain submodular functions as described in the full version of the paper would imply such a hardness result. What about hardness of solving integer linear programs with bounded minors?

- Of the major cases for our classification of Boolean CSPs, 2-SAT-MOD $M$ is special in that its complexity is not tied to the complexity of certain polynomial representations. Given that the polynomials representations which play a role for HORN-SAT-MOD $M$ and LIN-2-MOD $M$ are very closely related to the polymorphisms of HORN-SAT and LIN-2, AND and XOR respectively, how does the MAJ polymorphisms of
2-SAT play a role in the tractability of 2-SAT-MOD ${ }_{M}$ ? Understanding this would give us clues about how to generalize our results to CSPs over larger domains.

- Furthermore, 2-SAT-MOD $M$ is also the only case in which we lack a lower bound of the form $\Omega\left(n^{f(M)}\right)$, for some nontrivial function of $f$. Could it be that 2-SAT-MOD $M$ is fixedparameter tractable? For instance, does there exist a $2^{M}{ }_{n} O(1)$ algorithm? We note that the dependence on $M$ cannot be polynomial (unless $\mathrm{P}=\mathrm{NP}$ ), as setting $M$ greater than $n$ would solve 2-SAT with a global cardinality constraint, which is NP-complete.

- How do Mod-CSPs behave on a non-Boolean domain? Even for a domain of size three, the classification of ordinary CSPs is much more complex [6]. As such, in order for such a program to be carried out, one needs to better understand the interplay between the global modular constraints and the polymorphisms of these CSPs. In particular, how do notions like cores, bounded width, identities, etc., interplay with the modular constraints? See [3] for definitions of these terms.

- What other interesting global constraints can we impose on CSPs like 2-SAT, HORN-SAT, LIN-2, while still keeping them tractable? What happens if we add a global constraint over a non-Abelian group i.e. a global constraint of the form $\prod_{i=1}^{n} g_{i}^{x_{i}}=g_{0}$ for some $g_{0}, g_{1}, \ldots, g_{n} \in G$ where $G$ is a non-Abelian group.

\subsection{Organization}

In Section 2, we collect some basic facts about polynomials. We will present our algorithmic and hardness results for LIN-2-MOD ${ }_{M}$ in Section 3. The results for HORN-SAT-MOD $M$ and 2-SAT-MOD $M$ and the implications of our results to general Boolean Mod-CSPs can be found in the full version.

\section{PRELIMINARIES}

We will collect some useful lemmas about polynomial representations of modular constraints. The reader may skip these and come back when needed.

Lemma 2.1 (LuCAs's THeOREM). Let $p$ be a prime and let $a, b$ be non-negative integers with base $p$ expansions given by $a=a_{0}+a_{1} p+$ $a_{2} p^{2}+\ldots$ and $b=b_{0}+b_{1} p+b_{2} p^{2}+\ldots$ for some $0 \leqslant a_{i}, b_{i} \leqslant p-1$. Then

$$
\left(\begin{array}{l}
a \\
b
\end{array}\right)=\prod_{i \geqslant 0}\left(\begin{array}{l}
a_{i} \\
b_{i}
\end{array}\right) \quad \bmod p
$$

where $\left(\begin{array}{c}m \\ n\end{array}\right)=\frac{m(m-1) \ldots(m-(n-1))}{n !}$ for $n>0$ and $\left(\begin{array}{c}m \\ 0\end{array}\right)=1$ for all $m$.

Denote by $s_{k}(x)$ the $k^{t h}$ elementary symmetric polynomial given by $s_{k}(x)=\sum_{i_{1}<i_{2}<\cdots<i_{k}} x_{i_{1}} x_{i_{2}} \ldots x_{i_{k}}$.

LemMA 2.2. Let $p$ be some prime, $\ell \geqslant 1$ and $0 \leqslant a \leqslant p^{\ell}-1$ be integers. Let $a=a_{0}+a_{1} p+\cdots+a_{\ell-1} p^{\ell}$ be the base $p$ expansion of a. Then for every $x \in\{0,1\}^{n}$,

$\operatorname{Ham}(x)=a \quad \bmod p^{\ell} \Longleftrightarrow s_{p^{t}}(x)=a_{i} \quad \bmod p \forall 0 \leqslant t \leqslant \ell-1$.

Proof. Let $\operatorname{Ham}(x)=b_{0}+b_{1} p+b_{2} p^{2}+\ldots$ be the base $p$ expansion of $\operatorname{Ham}(x) \cdot \operatorname{Ham}(x)=a \bmod p^{\ell}$ iff $a_{t}=b_{t}$ for all $0 \leqslant t \leqslant \ell-1$. By Lemma 2.1, $\left(\begin{array}{c}\operatorname{Ham}(x) \\ p^{t}\end{array}\right)=\left(\begin{array}{c}b_{t} \\ 1\end{array}\right)=b_{t} \bmod p$. Note 
that $\left(\begin{array}{c}\operatorname{Ham}(x) \\ p^{t}\end{array}\right)=s_{p^{t}}(x)$. Thus $\operatorname{Ham}(x)=a \bmod p^{\ell}$ iff $s_{p^{t}}(x)=a_{t}$ $\bmod p$ for every $0 \leqslant t \leqslant \ell-1$.

Lemma 2.3. Let $p$ be some prime. For every $\ell \geqslant 1$ and $0 \leqslant a \leqslant$ $p^{\ell}-1$, there exists a degree $p^{\ell}-1$ polynomial $\phi_{\ell, a} \in \mathbb{F}_{p}\left[x_{1}, \ldots, x_{n}\right]$ such that for every $x \in\{0,1\}^{n}$,

$$
\phi_{\ell, a}(x) \quad \bmod p=\left\{\begin{array}{lll}
0 & \text { if } \operatorname{Ham}(x)=a & \bmod p^{\ell} \\
1 & \text { if } \operatorname{Ham}(x) \neq a & \bmod p^{\ell} .
\end{array}\right.
$$

Proof. Let $a=a_{0}+a_{1} p+\cdots+a_{\ell-1} p^{\ell-1}$ be the base $p$ expansion of $a$. By Lemma 2.2 and Fermat's little theorem,

$$
\phi_{\ell, a}(x)=1-\prod_{t=0}^{\ell-1}\left(1-\left(a_{t}-s_{p^{t}}(x)\right)^{p-1}\right)
$$

is the required polynomial of degree $(p-1)\left(1+p+\cdots+p^{\ell-1}\right)=$ $p^{\ell}-1$.

\section{$3 \quad$ LIN-2-MOD $M$}

A LIN-2 instance is a system of linear equations modulo 2 in $n$ variables $x_{1}, x_{2}, \ldots, x_{n} \in\{0,1\}$ i.e. each equation is of the form $\sum_{i} a_{i} x_{i}=a_{0}$ for some $a_{0}, a_{1}, \ldots, a_{n} \in\{0,1\}$. Satisfiability of a LIN-2 instance can be solved in polynomial time by Gaussian elimination.

Definition 3.1. LIN-2-MOD $M(n)$ is the following algorithmic problem: Given an instance of LIN-2 on $n$ variables $x_{1}, x_{2}, \ldots, x_{n}$ along with a modular constraint $\sum_{i} a_{i} x_{i}=a_{0} \bmod M$, decide if there is a solution in $x \in\{0,1\}^{n}$.

In this section, we will present an algorithm for LIN-2-MOD $M$ and analyze its running time.

\subsection{Algorithm for LIN-2-MOD $M$}

Wlog, we can assume that coefficients $a_{1}, \ldots, a_{n}=1$ in the modular constraint. This is because we can make $a_{i}$ copies of $x_{i}$ and add equality constraints among the copies. And equality is a LIN-2 constraint as $a=b$ iff $a \oplus b=0$. Since we can assume that original coefficients $a_{1}, \ldots, a_{n} \in 0,1, \ldots, M-1$, this increases the number of variables by a factor of $M$. Consider the following algorithm for this problem which depends on the parameter $R$, the number of rounds. We can calculate a basis for the set of solutions of a LIN-2 instance in polynomial time, so we will start with such a basis.

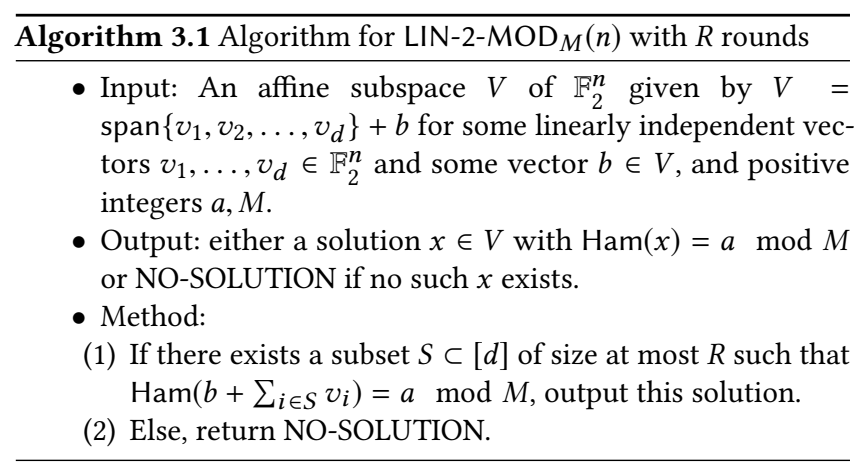

We will now prove that if we choose the number of rounds $R$ appropriately depending on $n, M$, then Algorithm 3.1 solves LIN-2-MOD $M(n)$ correctly. Since the running time of the algorithm is $O\left(n^{R}\right)$, the smaller the $R$ the better. Surprisingly, the value of $R$ required depends crucially on the prime factor decomposition of $M$ ! Let us start with a simple proposition which shows that if Algorithm 3.1 fails, then there should be a special kind of obstruction.

Proposition 3.2. If Algorithm 3.1 with $R$ rounds fails on an instance of $\operatorname{LIN}-2-\operatorname{MOD}_{M}(n)$, then there exists an affine subspace $U$ of $\mathbb{F}_{2}^{n}$ with dimension greater than $R$ with exactly one point $x^{*} \in U$ such that $\operatorname{Ham}\left(x^{*}\right)=a \bmod M$.

Proof. Suppose Algorithm 3.1 failed to find a solution after $R$ rounds. Therefore there exists a solution $x^{*}=b+\sum_{i \in S} v_{i}$ given a subset $S \subset[d]$ of size $|S|>R$ such that $\operatorname{Ham}\left(x^{*}\right)=a \bmod M$. Wlog, we can assume that $S$ has the minimum size among such sets. Now let $U$ be the affine subspace given by $U=b+\operatorname{span}\left\{v_{i}: i \in S\right\}$. The dimension of $U$ is $|S|$ which is greater than $R$. By minimality of $S$, every point in $y \in U$ other than $x^{*}$ has $\operatorname{Ham}(y) \neq a \bmod M . \quad \square$

Definition 3.3. Let $n, M$ be some positive integers. $\mathcal{D}(n, M)$ denotes the largest dimension of an affine subspace $C$ in $\mathbb{F}_{2}^{n}$ such that for some $0 \leqslant a \leqslant M$, there exists exactly one point $x_{0} \in C$ such that $\operatorname{Ham}\left(x_{0}\right)=a \bmod M$.

Therefore Proposition 3.2 implies the following corollary.

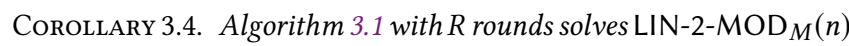
correctly if $R \geqslant \mathcal{D}(n, M)$.

The following proposition gives upper bounds on $\mathcal{D}(n, M)$ which in turn imply upper bounds on the number of rounds sufficient for our algorithm. The bounds depend crucially on the prime factor decomposition of $M$. Our bounds when $M$ is a prime power are nearly tight. The bound for general $M$ is conditional on a conjecture in additive combinatorics called the Polynomial Freiman-Ruzsa (PFR) conjecture, which we will define in Section 3.2.

Proposition 3.5. Let $n, M$ be positive integers. Then:

(1) $\mathcal{D}(n, M) \leqslant M-1$ if $M$ is a power of 2 .

(2) $\mathcal{D}(n, M) \leqslant(M-1) \log _{2}(n+1)$ if $M$ is an odd prime power.

(3) $\mathcal{D}(n, M) \leqslant 1+\left(M^{\prime}-1\right) \log _{2}(n+1)$ if $M=2 M^{\prime}$ for some odd prime power $M^{\prime}$.

(4) $\mathcal{D}(n, M) \lesssim_{\ell}\left(M^{\prime} \log n\right)^{2^{\ell-1}-1}$ if $M=2^{\ell} M^{\prime}$ for some odd prime power $M^{\prime}$ and $\ell \geqslant 2$ where $c>0$ is some absolute constant.

(5) $\mathcal{D}(n, M) \leqslant c_{M} n / \log n$ for some sufficiently large constant $c_{M}>0$ depending only on $M$ assuming the PFR conjecture.

We will prove Proposition 3.5 in Section 3.2 by a connection to sparsity of polynomials which represent $\mathrm{OR} \bmod M$ over $\{-1,1\}$ basis.

So when $M$ is a power of 2, we have a polynomial time algorithm

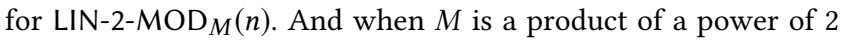
and an odd prime power, we have a quasipolynomial time algorithm. For general $M$, we have a slightly non-trivial running time of $\exp (O(n \log \log n / \log n))$, whereas the trivial algorithm which checks every solution takes $\exp (\Omega(n))$ time. By using randomization, we can considerably speed up the above algorithms. For this we make use the following proposition, which uses an amplification 
trick. It allows us to conclude that if there is one solution, then there should be many solutions.

Proposition 3.6. Let $V$ be an affine subspace of $\mathbb{F}_{2}^{n}$ and let

$$
N(V, a, M)=|\{x \in V: \operatorname{Ham}(x)=a \bmod M\}| .
$$

Then,

$$
N(V, a, M) \neq 0 \Rightarrow N(V, a, M) \geqslant \frac{|V|}{2^{\mathcal{D}(n, M)+1}} .
$$

Proof. Let $D=\mathcal{D}(n, M)+1$. Wlog we can assume that the dimension of $V$ is greater than $D$, otherwise the bound is trivially true. Since $N(V, a, M) \neq 0$, we can find some $x^{*} \in V$ such that $\operatorname{Ham}\left(x^{*}\right)=a \bmod M$. Let $T \subset V \backslash\left\{x^{*}\right\}$ be the set of all points in $y \in V \backslash\left\{x^{*}\right\}$ such that $\operatorname{Ham}(y)=a \bmod M$. Pick a random affine subspace $A$ inside $V$ of dimension $D$ passing through $x^{*}$. By the definition of $\mathcal{D}(n, M)$, there exists an other point $z \in A \backslash\left\{x^{*}\right\}$ such that $\operatorname{Ham}(z)=a \bmod M$. Therefore $|T \cap A| \geqslant 1$. Therefore,

$$
\begin{aligned}
1 & \leqslant \mathbb{E}_{A}[|T \cap A|] \\
& =\mathbb{E}_{A}\left[\sum_{x \in V \backslash\left\{x^{*}\right\}} \mathbb{1}_{T}(x) \mathbb{1}_{A}(x)\right] \\
& =\sum_{x \in V \backslash\left\{x^{*}\right\}} \mathbb{1}_{T}(x) \mathbb{E}_{A}\left[\mathbb{1}_{A}(x)\right] \\
& =\sum_{x \in V \backslash\left\{x^{*}\right\}} \mathbb{1}_{T}(x) \frac{2^{D}-1}{|V|-1}=|T| \frac{2^{D}-1}{|V|-1} .
\end{aligned}
$$

Therefore $N(V, a, M)=1+|T| \geqslant 1+\frac{|V|-1}{2^{D}-1} \geqslant \frac{|V|}{2^{D}}$.

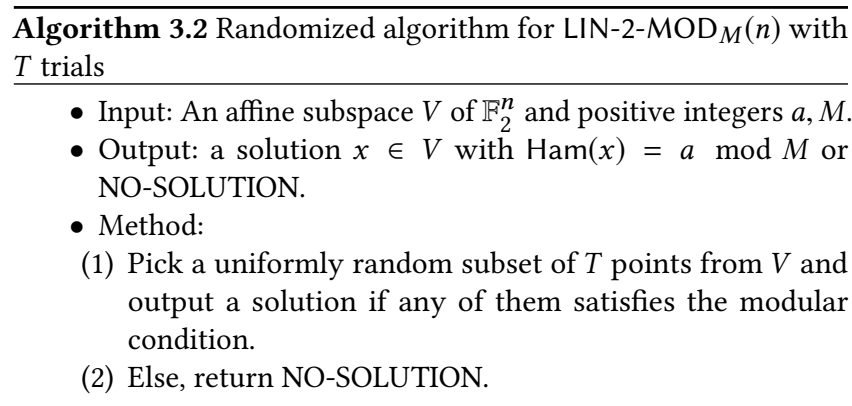

Combining Proposition 3.5 with Proposition 3.6 we get the following corollary.

Corollary 3.7. Algorithm 3.2 with T trials outputs correctly given an instance of $\operatorname{LIN}-2-\mathrm{MOD}_{M}(n)$ with probability at least $2 / 3$ if $T \geqslant$ $4 \cdot 2^{\mathcal{D}(n, M)}$.

Proof. By Proposition 3.6, if there exists a solution, then Algorithm 3.2 will find it with probability

$1-\left(1-1 / 2^{\mathcal{D}(n, M)+1}\right)^{T} \geqslant 1-e^{-T / 2^{\mathcal{D}(n, M)+1}} \geqslant 1-e^{-2} \geqslant 2 / 3$.

\subsection{Sparsity of Polynomials Representing $\mathrm{OR}_{d}$ $\bmod M$ over $\{-1,1\}^{d}$}

In this section, we will prove the upper bounds on $\mathcal{D}(n, M)$ stated in Proposition 3.5 by a reduction to understanding the sparsity of polynomials which represent OR $\bmod M$ over $\{-1,1\}$ basis (this is presented in Proposition 3.10). For simplicity, we will assume throughout that $M$ is odd. Upper bounds on $\mathcal{D}(n, M)$ when $M$ is even can be obtained by a reduction to odd $M$ (see the full version for details).

3.2.1 Reduction of $\mathcal{D}(n, M)$ to Sparsity of OR $\bmod M$ over $\{-1,1\}$ Basis for Odd M. Let's start with the definition of a polynomial representation of OR $\bmod M$ over $\{-1,1\}$ basis.

Definition 3.8. A polynomial $p\left(x_{1}, \ldots, x_{d}\right)$ is said to represent $\mathrm{OR}_{d} \bmod M$ over $\{-1,1\}^{d}$ if it has integer coefficients and

$$
p(x)\left\{\begin{array}{lll}
= & 0 & \bmod M \text { if } x=1 \\
\neq & 0 & \bmod M \text { if } x \in\{-1,1\}^{d} \backslash\{\mathbf{1}\}
\end{array}\right.
$$

where 1 is the all ones vector.

To make the required reduction, we need the following proposition which relates the Hamming weights of points in a $d$-dimensional affine subspace to evaluations of a polynomial over $\{-1,1\}^{d}$. For $\mathbf{a}=$ $\left(a_{1}, \ldots, a_{\ell}\right) \in \mathbb{F}_{2}^{\ell}$, let $(-1)^{\mathrm{a}}$ denote the vector $\left((-1)^{a_{1}}, \ldots,(-1)^{a_{\ell}}\right)$. Given a polynomial $p$ with integer coefficients, define $|p|$ as sum of the absolute value of its coefficients. Note that the number of monomials in $p$ is always at most $|p|$.

Proposition 3.9. Let $\mathbf{b}, \mathbf{u}_{1}, \ldots, \mathbf{u}_{d} \in \mathbb{F}_{2}^{n}$. Then there exists a multilinear polynomial $p\left(z_{1}, \ldots, z_{d}\right)$ with integer coefficients and $|p|=n$ such that for every $\mathbf{y} \in \mathbb{F}_{2}^{d}$,

$$
n-2 \operatorname{Ham}\left(\mathbf{b}+\sum_{i} y_{i} \mathbf{u}_{i}\right)=p\left((-1)^{\mathbf{y}}\right) .
$$

Conversely, given any multilinear polynomial $p\left(z_{1}, \ldots, z_{d}\right)$ with integer coefficients and $|p|=n$, there exists $\mathbf{b}, \mathbf{u}_{1}, \ldots, \mathbf{u}_{d} \in \mathbb{F}_{2}^{n}$ satisfying the above identity.

Proof. We will first start with $\mathbf{b}, \mathbf{u}_{1}, \ldots, \mathbf{u}_{d} \in \mathbb{F}_{2}^{n}$ and construct such a polynomial $p$. Let $\mathbf{b}=\left(b_{1}, \ldots, b_{n}\right)$ and $\mathbf{u}_{i}=\left(u_{i 1}, \ldots, u_{i n}\right)$. Define the map $\phi:\{-1,1\}^{d} \rightarrow\{-1,1\}^{n}$ as:

$$
\phi_{t}\left(z_{1}, \ldots, z_{d}\right)=(-1)^{b_{t}} \prod_{i \in[d]: u_{i t}=1} z_{i}
$$

Then,

$$
\begin{aligned}
\phi_{t}\left((-1)^{\mathrm{y}}\right) & =(-1)^{b_{t}} \prod_{i \in[d]: u_{i t}=1}(-1)^{y_{i}} \\
& =(-1)^{b_{t}} \prod_{i \in[d]}(-1)^{y_{i} u_{i t}} \\
& =(-1)^{b_{t}+\sum_{i \in[d]} y_{i} u_{i t}} .
\end{aligned}
$$

Therefore for any $\mathbf{y} \in \mathbb{F}_{2}^{d}, \phi\left((-1)^{\mathrm{y}}\right)=(-1)^{\mathbf{b}+\sum_{i=1}^{d} y_{i} \mathbf{u}_{i}}$. Define $p$ as: $p(z)=\sum_{t=1}^{n} \phi_{t}(z)$. Note that $p$ has integer coefficients and $|p|=n$. And finally,

$\operatorname{Ham}\left(\mathbf{b}+\sum_{i \in[d]} y_{i} \mathbf{u}_{i}\right)=\sum_{t=1}^{n} \frac{1}{2}\left(1-\phi_{t}\left((-1)^{\mathbf{y}}\right)\right)=\frac{1}{2}\left(n-p\left((-1)^{\mathbf{y}}\right)\right)$. 
To prove the converse, we just execute the steps of the above construction in reverse. Given a polynomial $p\left(z_{1}, \ldots, z_{d}\right)$ with integer coefficients and $|p|=n$, let $\phi_{1}(z), \ldots, \phi_{n}(z)$ be (signed, possibly repeated) monomials in $z$ be such that $p(z)=\sum_{t=1}^{n} \phi(z)$. Now define $\mathbf{b}, \mathbf{u}_{1}, \ldots, \mathbf{u}_{d} \in \mathbb{F}_{2}^{n}$ such that $\phi_{t}(z)=(-1)^{b_{t}} \prod_{i \in[d]: u_{i t}=1} z_{i}$ is true, explicitly

$$
u_{i t}=\left\{\begin{array}{ll}
1 & \text { if } \phi_{t}(z) \text { contains } z_{i} \\
0 & \text { else }
\end{array} .\right.
$$

Then by the same argument as above, the required identity is satisfied.

The following proposition shows the connection between affine subspaces which are obstructions for Algorithm 3.1 and polynomials representing OR $\bmod M$ over $\{-1,1\}$ basis.

Proposition 3.10. Suppose $M$ is odd. Let $d=\mathcal{D}(n, M)$, then there exists a polynomial $f\left(x_{1}, \ldots, x_{d}\right)$ with at most $n+1$ monomials that represents $\mathrm{OR}_{d} \bmod M$ over $\{-1,1\}^{d}$. Conversely, given a polynomial with $n$ monomials which represents $\mathrm{OR}_{d} \bmod M$ over $\{-1,1\}^{d}$, $\mathcal{D}\left(n^{\prime}, M\right) \geqslant d$ for some $n^{\prime} \leqslant M \cdot n$.

Proof. Suppose $U$ is a $d$-dimensional affine subspace of $\mathbb{F}_{2}^{n}$ which contains exactly one point $x^{*} \in U$ such that $\operatorname{Ham}(x)=a$ $\bmod M$.

Let $\mathbf{b}, \mathbf{u}_{1}, \ldots, \mathbf{u}_{d} \in \mathbb{F}_{2}^{n}$ be such that $U=\mathbf{b}+\operatorname{span}\left\{\mathbf{u}_{1}, \ldots, \mathbf{u}_{d}\right\}$. By Proposition 3.9, there exists a polynomial $p\left(z_{1}, \ldots, z_{d}\right)$ with integer coefficients and at most $n$ monomials such that for every $\mathbf{y} \in \mathbb{F}_{2}^{d}$, $n-2 \operatorname{Ham}\left(\mathbf{b}+\sum_{i=1}^{d} y_{i} \mathbf{u}_{i}\right)=p\left((-1)^{\mathbf{y}}\right)$. Suppose $x^{*}=\mathbf{b}+\sum_{i} y_{i}^{*} \mathbf{u}_{i}$, then $p(z)=(n-2 a) \bmod M$ for exactly one $z$ in $\{-1,1\}^{d}$ given by $z=z^{*}=(-1)^{y^{*}}$ (here we are using the fact that $M$ is odd).

Define the polynomial $f(z)=p\left(z \odot z^{*}\right)-(n-2 a)$ where $z \odot z^{*}$ is the coordinate wise product. Note that $f(\mathbf{1})=0 \bmod M$ and $f(z) \neq 0$ $\bmod M$ for all $z \in\{-1,1\}^{d} \backslash\{\mathbf{1}\}$. Thus $f(z)$ is a polynomial which represents $\mathrm{OR}_{d} \bmod M$ over $\{-1,1\}^{d}$ and $f$ has at most $n+1$ monomials.

To prove the converse, suppose $f(z)$ is a polynomial with $n$ monomials which represents $\mathrm{OR}_{d} \bmod M$ over $\{-1,1\}^{d}$. Wlog, we can assume that the coefficients of $f$ are in $\{0,1, \ldots, M-1\}$. Let $n^{\prime}=|f|$, by our assumption about coefficients of $f, n^{\prime} \leqslant M n$. By the converse part in Proposition 3.9, there exists $\mathbf{b}, \mathbf{u}_{1}, \ldots, \mathbf{u}_{d} \in \mathbb{F}_{2}^{n^{\prime}}$, such that $n^{\prime}-2 \operatorname{Ham}\left(\mathbf{b}+\sum_{i=1}^{d} y_{i} \mathbf{u}_{i}\right)=f\left((-1)^{\mathbf{y}}\right)$ for every $\mathbf{y} \in \mathbb{F}_{2}^{d}$. Note that $\mathbf{u}_{1}, \ldots, \mathbf{u}_{d}$ must be linearly independent. If not, there exists a $\mathbf{y} \neq 0$ such that $f\left((-1)^{\mathbf{y}}\right)=f(1)=0 \bmod M$ which is a contradiction.

Therefore in the $d$-dimensional affine subspace given by $V=\{\mathbf{b}+$ $\left.\sum_{i=1}^{n^{\prime}} y_{i} \mathbf{u}_{i}: \mathbf{y} \in \mathbb{F}_{2}^{d}\right\}$, there exists exactly one point $\mathbf{x}^{*} \in V$ (given by $\left.\left.\mathbf{x}^{*}=\mathbf{b}\right)\right)$ such that $\operatorname{Ham}\left(\mathbf{x}^{*}\right)=(n / 2) \bmod M$. Thus $\mathcal{D}\left(n^{\prime}, M\right) \geqslant d$.

Because of the above proposition, if we prove sparsity lower bounds on polynomials which represent $O R \bmod M$ then we get good upper bounds on the number of rounds that will be enough in Algorithm 3.1.

3.2.2 When $M$ is an Odd Prime Power. Now we will show that when $M$ is an odd prime power, a polynomial which represents
$\mathrm{OR}_{d} \bmod M$ over $\{-1,1\}^{d}$ should have exponential number of monomials. We will collect some facts that we need to prove this.

Proposition 3.11. Ifp is an odd prime, any function $f:\{-1,1\}^{d} \rightarrow$ $\mathbb{F}_{p}$ has a unique representation as a multilinear polynomial.

The following lemma explicitly gives the polynomial which calculates $\mathrm{OR}_{d} \bmod p$ exactly.

Proposition 3.12. Suppose a multilinear polynomial $f\left(z_{1}, \ldots, z_{d}\right)$ exactly represents $\mathrm{OR}_{d}$ mod $p$ over $\{-1,1\}^{d}$ for some odd prime $p$ i.e.

$$
f(z)\left\{\begin{array}{lll}
= & 0 & \bmod p \text { if } z=1 \\
= & 1 & \bmod p \text { if } x \in\{-1,1\}^{d} \backslash\{1\}
\end{array} .\right.
$$

Then $f(z)$ has $2^{d}$ monomials and explicitly given by,

$$
f(z)=1-\prod_{i \in[d]} \frac{\left(1+z_{i}\right)}{2}
$$

The following proposition provides a sparsity lower bound when $M$ is an odd prime power.

Proposition 3.13. Suppose $M$ is an odd prime power. If a polynomial $f(z)$ represents $\mathrm{OR}_{d} \bmod M$ over $\{-1,1\}^{d}$, then $f$ has at least $2^{d /(M-1)}$ monomials.

Proof. Let $M=p^{k}$ for some odd prime $p$. Wlog, we can assume that the coefficients of $f$ are in $\{0,1, \ldots, M-1\}$. Let $\Psi_{f}^{\prime}(z)$ be the vector of monomials of $f$ evaluated at $z$, where each monomial appears with multiplicity equal to its coefficient in $f$, and let $N$ be its length. For $z \in\{-1,1\}^{d}, \Psi_{f}^{\prime}(z) \in\{-1,1\}^{N}$ and $f(z)=$ $\sum_{i \in[N]}\left(\Psi_{f}^{\prime}(z)\right)_{i}$. Let $\Psi_{f}(z)$ be the vector of the same length as $\Psi_{f}^{\prime}(z)$ whose coordinates are given by

$$
\left(\Psi_{f}(z)\right)_{i}=\frac{1+\left(\Psi_{f}^{\prime}(z)\right)_{i}}{2} .
$$

For $z \in\{-1,1\}^{d}, \Psi_{f}(z) \in\{0,1\}^{N}$ and

$$
\operatorname{Ham}\left(\Psi_{f}(z)\right)=\sum_{i=1}^{N} \frac{1+\left(\Psi_{f}^{\prime}(z)\right)_{i}}{2}=\frac{N}{2}+\frac{f(z)}{2} .
$$

Therefore for $z \in\{-1,1\}^{d}, \operatorname{Ham}\left(\Psi_{f}(z)\right)=N / 2 \bmod p^{k}$ iff $z=1$.

By Lemma 2.3, there exists a polynomial $\phi$ of degree $p^{k}-1=M-1$ such that

$$
\phi\left(\Psi_{f}(z)\right)=\left\{\begin{array}{lll}
0 & \bmod p \text { if } \operatorname{Ham}\left(\Psi_{f}(z)\right)=N / 2 & \bmod p^{k} \\
1 & \bmod p \text { if } \operatorname{Ham}\left(\Psi_{f}(z)\right) \neq N / 2 & \bmod p^{k}
\end{array} .\right.
$$

Therefore $\phi\left(\Psi_{f}(z)\right)$ exactly represents $\mathrm{OR}_{d} \bmod p$ over $\{-1,1\}^{d}$ and therefore by Proposition 3.12, it has $2^{d}$ monomials. Since $\phi$ has degree $M-1$, the number of monomials in $\phi\left(\Psi_{f}(z)\right)$ is at most $\left(\begin{array}{c}N^{\prime} \\ \leqslant M-1\end{array}\right)$ where $N^{\prime}$ is the number of (distinct) monomials in $f$. Therefore, $N^{\prime} \geqslant 2^{d /(M-1)}$.

Thus we have the following corollary which proves part (2) of Proposition 3.5.

Corollary 3.14. Let $M$ be an odd prime power. Then $\mathcal{D}(n, M) \leqslant$ $(M-1) \log _{2}(n+1)$. 
Proof. Suppose $C$ is an affine subspace of $\mathbb{F}_{2}^{n}$ of dimension $d>(M-1) \log _{2}(n+1)$ which contains exactly one point $x_{0}$ such that $\operatorname{Ham}\left(x_{0}\right)=a \bmod M$. Then by Proposition 3.9, there exists a polynomial $p\left(x_{1}, \ldots, x_{d}\right)$ with at most $n+1$ monomials such that $p(x)$ represents $O R_{d} \bmod M$ over $\{-1,1\}^{d}$. Therefore by Proposition $3.13,(n+1) \geqslant 2^{d /(M-1)}$ which is a contradiction.

We remark that the above bound is nearly tight. Let $n=(M-1) 2^{d}$. The Hadamard code is a subspace of $\mathbb{F}_{2}^{2}$ of dimension $d$ such that every non-zero point in the subspace has weight $2^{d-1}$. Decompose $\mathbb{F}_{2}^{n}=\bigoplus \mathbb{F}_{2}^{2^{d}}$ where the copies of $\mathbb{F}_{2}^{2^{d}}$ are supported on mutually disjoint sets of variables. Let $V$ be the subspace of $\mathbb{F}_{2}^{n}$ which is the direct sum of Hadamard codes in each copy of $\mathbb{F}_{2}^{2}$. Then $V$ has dimension $(M-1) d=(M-1) \log _{2}(n /(M-1))$ and every non-zero point in $V$ has weight in $\left\{1 \cdot 2^{d-1}, 2 \cdot 2^{d-1}, \ldots,(M-1) \cdot 2^{d-1}\right\}$ which is non-zero modulo $M$.

3.2.3 When $M$ has Multiple Odd Prime Factors. We will now focus on the case when $M$ is odd and has multiple prime factors. Unfortunately, in this case we do not know any unconditional super linear lower bounds on the sparsity of polynomials representing $\mathrm{OR}_{d}$ $\bmod M$ over $\{-1,1\}^{d}$. But we can get a conditional super linear lower bound, assuming Polynomial Freiman-Ruzsa (PFR) conjecture which is a well-known conjecture in additive combinatorics. We achieve this by constructing matching vector families in $(\mathbb{Z} / M \mathbb{Z})$ starting from sparse representations of $\mathrm{OR}_{d} \bmod M$ over $\{-1,1\}^{d}$. We will first define matching vector families.

Definition 3.15. A matching vector family (MVF) over $\mathbb{Z} / M \mathbb{Z}$ of rank $r$ and size $N$ is a collection of vectors $\mathbf{u}_{1}, \ldots, \mathbf{u}_{N} \in(\mathbb{Z} / M \mathbb{Z})^{r}$ and $\mathbf{v}_{1}, \ldots, \mathbf{v}_{N} \in(\mathbb{Z} / M \mathbb{Z})^{r}$ such that for every $i, j \in[N]$ :

$$
\left\langle\mathbf{u}_{i}, \mathbf{v}_{j}\right\rangle \begin{cases}=0 & \bmod M \text { if } i=j \\ \neq 0 & \bmod M \text { if } i \neq j .\end{cases}
$$

MVFs over $\mathbb{Z} / M \mathbb{Z}$ of low rank and large size have found applications in many areas. They are used in the construction of constant query locally decodable codes $[13,15,30]$, Ramsey graphs [18, 19], private information retrieval schemes [14] and secret sharing schemes [23]. In particular, this implies that lower bounds for constant query locally decodable codes give lower bounds on the rank of MVFs of a given size. For example, super polynomial lower bounds on the length of constant query locally decodable codes imply that the sparsity of a polynomial representing $\mathrm{OR}_{d} \bmod M$ over $\{-1,1\}^{d}$ should be $\omega(d)$. But only polynomial lower bounds on constant query locally decodable codes are known $[21,22]^{7}$. In fact, we do not even know any strong unconditional lower bounds on the rank of MVFs over $\mathbb{Z} / M \mathbb{Z}$ i.e. results of the form $N \leqslant \exp \left(o_{M}(r)\right)$. But assuming the PFR conjecture, the following bound is known. We will not state the PFR conjecture here, for the precise statement see [4].

Proposition 3.16 ([4]). Assuming the Polynomial Freiman-Ruzsa conjecture over $(\mathbb{Z} / M \mathbb{Z})^{r}$, any $M V F$ over $\mathbb{Z} / M \mathbb{Z}$ of rank $r$ should have size $N \leqslant \exp \left(O_{M}(r / \log r)\right)$.

\footnotetext{
${ }^{7}$ For 2-query locally decodable codes, it is known that the length of the encoding should be exponential in the message length [22]. But for $q \geqslant 3$, the best lower bounds are only polynomial.
}

We are now ready to prove the super linear lower bound on the sparsity of polynomials representing $\mathrm{OR}_{d} \bmod M$ over $\{-1,1\}^{d}$ assuming PFR.

Proposition 3.17. Assuming the Polynomial Freiman-Ruzsa (PFR) conjecture in $(\mathbb{Z} / M \mathbb{Z})^{d}$, any polynomial that represents $\mathrm{OR}_{d} \bmod M$ over $\{-1,1\}^{d}$ needs to have $\Omega_{M}(d \log d)$ monomials.

Proof. Given a sparse polynomial that represents $\mathrm{OR}_{d} \bmod M$ over $\{-1,1\}^{d}$, we will construct a MVF over $\mathbb{Z} / M \mathbb{Z}$ of small rank and large size. The construction is a based on a similar construction due to Sudan which first appeared in [17]. Suppose $p(z)=$ $\sum_{t=1}^{r} a_{t} \prod_{i \in S_{t}} z_{i}$ is a polynomial with $r$ monomials which represents $\mathrm{OR}_{d} \bmod M$ over $\{-1,1\}^{d}$. Define the $2^{d} \times 2^{d}$ matrix $A$ with rows and columns indexed by $\{-1,1\}^{d}$ as

$$
A\left(z, z^{\prime}\right)=p\left(z \odot z^{\prime}\right) \quad \bmod M
$$

where $z \odot z^{\prime}$ is the component-wise product. Note that the diagonal entries

$$
A(z, z)=p(z \odot z)=p(1)=0 \quad \bmod M
$$

and the off-diagonal entries are

$$
A\left(z, z^{\prime}\right)=p\left(z \odot z^{\prime}\right) \neq 0 \quad \bmod M .
$$

Moreover the rank of the matrix $A$ is at most $r$ since

$A\left(z, z^{\prime}\right)=p\left(z \odot z^{\prime}\right)=\sum_{t} a_{t} \prod_{i \in S_{t}} z_{i} z_{i}^{\prime}=\left\langle\left(a_{t} \prod_{i \in S_{t}} z_{i}\right)_{t},\left(\prod_{i \in S_{t}} z_{i}^{\prime}\right)_{t}\right\rangle$.

Therefore the set of vectors $\mathbf{u}_{z}=\left(a_{t} \prod_{i \in S_{t}} z_{i}\right)_{t \in[r]} \in(\mathbb{Z} / M \mathbb{Z})^{r}$ and $\mathbf{v}_{z^{\prime}}=\left(\prod_{i \in S_{t}} z_{i}^{\prime}\right)_{t \in[r]} \in(\mathbb{Z} / M \mathbb{Z})^{r}$ for $z, z^{\prime} \in\{-1,1\}^{d}$ form a MVF over $(\mathbb{Z} / M \mathbb{Z})$ of size $N=2^{d}$ of rank $r$. By Proposition 3.16,

$$
\begin{aligned}
N \leqslant \exp \left(O_{M}(r / \log r)\right) & \Rightarrow d \leqslant O_{M}(r / \log r) \\
& \Rightarrow r \geqslant \Omega_{M}(d \log d) .
\end{aligned}
$$

So we have the following corollary which proves part (5) of Proposition 3.5.

Corollary 3.18. Assuming the PFR conjecture, for every positive integer $M$, there exists a constant $c_{M}$ depending only on $M$ such that, $\mathcal{D}(n, M) \leqslant c_{M} n / \log n$.

Proof. Suppose $C$ is an affine subspace of $\mathbb{F}_{2}^{n}$ of dimension $d>c_{M} n / \log n$ which contains exactly one point $x_{0}$ such that $\operatorname{Ham}\left(x_{0}\right)=a \bmod M$. Then by Proposition 3.9, there exists a polynomial $p\left(x_{1}, \ldots, x_{d}\right)$ with at most $n+1$ monomials such that $p(x)$ represents $O R_{d} \bmod M$ over $\{-1,1\}^{d}$. Therefore by Proposition 3.17, $(n+1) \geqslant \Omega_{M}(d \log d)$ which is a contradiction if we choose $c_{M}$ sufficiently large.

\subsection{Hardness of LIN-2-MOD $M$}

We will now show hardness for LIN-2-MOD $M$ when $M$ has multiple odd prime factors assuming exponential time hypothesis (ETH). We will show that if there are low degree polynomials representing $\mathrm{OR}_{d} \bmod M$, then solving $\mathrm{LIN}-2-\mathrm{MOD}_{M}$ is hard.

Proposition 3.19. Let $M$ be odd and suppose $f(\cdot)$ is some function such that for every $d$, there exists a degree $f(d)$ polynomial which represents $\mathrm{OR}_{d} \bmod M$ over $\{-1,1\}^{d}$ which is efficiently computable. Then assuming ETH, solving $\mathrm{LIN}-2-\mathrm{MOD}_{M}(n)$ requires at 
least $2^{\Omega(m)}-\operatorname{poly}(n)$ time for some $m$ such that $f(m) \log (m / f(m)) \gtrsim$ $\log (n)$.

Proof. Choose the largest $m$ such that $\left(\begin{array}{c}3 m \\ \leqslant 3 f(m)\end{array}\right) \leqslant n$, such an $m$ will satisfy $f(m) \log (m / f(m)) \gtrsim \log (n)$. Suppose $\phi(x)=$ $C_{1}(x) \wedge C_{2}(x) \wedge \cdots \wedge C_{m}(x)$ is some 3-SAT instance with $m$ clauses and $t \leqslant 3 m$ variables where each $C_{i}(x)$ depends on at most 3 variables. We can assume that the variables $x_{1}, \ldots, x_{t}$ take $\{-1,1\}$ values and each $C_{i}(x)$ is a polynomial which takes these $\{-1,1\}$ values and outputs 1 if the $i^{t h}$ clause is satisfied and -1 if it is not. So $\phi$ is satisfiable iff there exists some $x \in\{-1,1\}^{t}$ such that $C_{1}(x)=\cdots=C_{m}(x)=1$. Now let $p\left(z_{1}, \ldots, z_{m}\right)$ be a polynomial of degree $f(m)$ which represents $\mathrm{OR}_{m} \bmod M$. Then $\phi$ is satisfiable iff there exists some $x \in\{-1,1\}^{t}$ such that the polynomial $\Psi(x)=p\left(C_{1}(x), \ldots, C_{m}(x)\right)=0 \bmod M$. The polynomial $\Psi$ has degree at most $3 f(m)$ and so it has at most $\left(\begin{array}{c}t \\ \leqslant 3 f(m)\end{array}\right) \leqslant n$ monomials. Wlog we can assume that $\Psi$ has coefficients in $\{1,2, \ldots, M-1\}$ because we only care about its values modulo $M$, let us denote these coefficients by $a_{1}, a_{2}, \ldots, a_{n}$. Emulating the proof of Proposition 3.9, there exists $\mathbf{u}_{1}, \ldots, \mathbf{u}_{t} \in \mathbb{F}_{2}^{n}$ (which can be computed efficiently from $\Psi$ ) such that for every $\mathrm{y} \in \mathbb{F}_{2}^{t}$,

$\Psi\left((-1)^{\mathbf{y}}\right)=\sum_{j=1}^{n} a_{j}\left(1-2\left(\sum_{i=1}^{t} y_{i} \mathbf{u}_{i}\right)_{j}\right)=\sum_{j=1}^{n} a_{j}-2 \sum_{j=1}^{n} a_{j}\left(\sum_{i=1}^{t} y_{i} \mathbf{u}_{i}\right)_{j}$.

Therefore $\Psi(x)=0 \bmod M$ for some $x \in\{-1,1\}^{t}$ iff there exists some $\mathbf{x}^{\prime} \in \operatorname{span}\left\{\mathbf{u}_{1}, \ldots, \mathbf{u}_{t}\right\}$ such that $\sum_{j=1}^{n} a_{j}\left(\mathbf{x}^{\prime}\right)_{j}=\left(\sum_{j=1}^{n} a_{j}\right) / 2$ $\bmod M$. We can write the condition $\mathbf{x}^{\prime} \in \operatorname{span}\left\{\mathbf{u}_{1}, \ldots, \mathbf{u}_{t}\right\}$ as a system of linear equations over $\mathbb{F}_{2}$ that $\mathbf{x}^{\prime}$ should satisfy, explicitly, $U^{\perp} \mathbf{x}^{\prime}=\overline{0}$ where $U^{\perp}$ is the matrix whose rows form a basis for the orthogonal complement of $\operatorname{span}\left\{\mathbf{u}_{1}, \mathbf{u}_{2}, \ldots, \mathbf{u}_{t}\right\}$.

Thus we reduced an instance of 3-SAT with $m$ clauses to an instance of LIN-2-MOD $M(n)$. The reduction itself takes poly $(n)$ time. By ETH, 3-SAT requires $2^{\Omega(m)}$ time. This proves that we need $2^{\Omega(m)}-\operatorname{poly}(n)$ time to solve LIN-2-MOD $M(n)$.

Remark: Note that the gadgets we used in the hardness proof are low-degree polynomials which represent OR $\bmod M$ over $\{-1,1\}$ basis. Whereas the obstructions to our algorithm are sparse polynomials which represent $\mathrm{OR} \bmod M$ over $\{-1,1\}$ basis. It is tempting to believe that the obstructions to the optimal algorithm should be the right gadgets that should be used in the hardness proof. Here is a different reduction. Start with a GAP-3LIN instance $\phi(x)=\left(E_{1}(x), \ldots, E_{m}(x)\right)$ over $t$ variables where it is promised that either $1-\varepsilon$ fraction of equations are satisfiable or less than $1 / 2+\varepsilon$ fraction are satisfiable. GAP-3LIN is NP-hard and there are near-linear time reductions from 3-SAT to GAP-3LIN [24]. Suppose $p\left(z_{1}, \ldots, z_{m}\right)$ is a polynomial over $\{-1,1\}^{m}$ such that it weakly represents this (partial) threshold function modulo $M$ That is the values of $p(z) \bmod M$ when $\sum_{i} z_{i} \geqslant(1-2 \varepsilon) m$ and when $\sum_{i} z_{i} \leqslant 2 \varepsilon m$ are disjoint, say $S_{1}$ and $S_{0}$ respectively. Then $\phi$ is $(1-\varepsilon)$-satisfiable iff there exists some $x \in\{-1,1\}^{t}$ such that $\Psi(x)=p\left(E_{1}(x), \ldots, E_{m}(x)\right) \in S_{1}$. But note that in the $\{-1,1\}$ basis, the sparsity of $\Psi$ is the same as sparsity of $p$. Thus we get a good hardness reduction if there are sparse polynomials which weakly represent the $(1-\varepsilon, 1 / 2+\varepsilon)$-threshold partial function. Another interesting question is, can we use the non-existence of such sparse polynomials in creating a good algorithm for $\mathrm{LIN}^{-2-\mathrm{MOD}_{M}}$ ?

Proposition 3.20. For any odd integer $M \geqslant 2$, there exists $a$ degree $\lceil d /(M-1)\rceil$ polynomial which represents $\mathrm{OR}_{d} \bmod M$ over $\{-1,1\}^{d}$.

Proof. Partition the variables $x_{1}, x_{2}, \ldots, x_{d}$ into $M-1$ parts of size at most $d^{\prime}=\lceil d /(M-1)\rceil$. We can compute the OR of each part exactly with a degree $d^{\prime}$ polynomial of the form 1 $\prod_{i=1}^{d^{\prime}}\left(\frac{1+x_{i}}{2}\right)$. Note that powers of 2 in the denominator can be inverted $\bmod M$ to get a polynomial with integer coefficients. Adding these polynomials which compute OR on each part exactly, we get a polynomial which represents $\mathrm{OR}_{d} \bmod M$ over $\{-1,1\}^{d}$.

We have shown that LIN-2-MOD $M(n)$ can be solved in randomized time $n^{M+O(1)}$ when $M$ is an odd prime power. Combining Propositions 3.20 and 3.19 we have the following corollary, which shows that our this running time is nearly tight assuming ETH when $M$ is an odd prime power.

Corollary 3.21. Suppose $3 \leqslant M \leqslant c n$ be an odd integer for some small enough constant $0<c<1$. Assuming ETH, solving LIN-2-MOD $M(n)$ requires at least $n^{\Omega(M / \log M)}$ time.

The following proposition by Barrington, Beigel and Rudich [2], from the paper where they first introduced polynomial representations modulo composites, shows that there are non-trivial low degree polynomials representing OR $\bmod M$ if $M$ has multiple prime factors.

Proposition 3.22 ([2]). Suppose $M$ has $r$ distinct prime factors. There exists an explicit degree $O_{M}\left(t^{1 / r}\right)$ polynomial which represents $\mathrm{OR}_{t} \bmod M$ over $\{-1,1\}^{t}$. Moreover it can computed in time polynomial in its size.

This immediately implies hardness of LIN-2-MOD $M(n)$ when $M$ has multiple odd prime factors.

Corollary 3.23. Suppose $M$ has $r$ distinct odd prime factors. Assuming ETH, solving LIN-2-MOD $M(n)$ requires at least $\exp \left(\Omega_{M}\left((\log n / \log \log n)^{r}\right)\right)$ time.

Proof. Let $M=2^{\ell} M^{\prime}$ for some odd $M^{\prime}$. Since we can reduce $\mathrm{LIN}-2-\mathrm{MOD}_{M^{\prime}}(n)$ to LIN-2-MOD $M(n)$ easily, it is enough to show hardness for LIN-2-MOD $M^{\prime}(n)$. So wlog, we can assume $M$ is odd.

When $M$ is odd, a polynomial which represents NAND over $\{0,1\}$ basis can be converted into a polynomial with represents OR over $\{-1,1\}$ basis by a linear basis change which preserves the degree. Therefore by Proposition 3.22, we can take $f(m)=$ $O_{M}\left(m^{1 / r}\right)$ in Proposition 3.19. $m^{1 / r} \log m=\Omega_{M}(\log n)$ implies that

$$
m \gtrsim_{M}\left(\frac{\log n}{\log \log n}\right)^{r}
$$

which implies the required bound. 


\section{REFERENCES}

[1] Stephan Artmann, Robert Weismantel, and Rico Zenklusen. 2017. A strongly polynomial algorithm for bimodular integer linear programming. In Proceedings of the 49th Annual ACM SIGACT Symposium on Theory of Computing. ACM, 1206-1219.

[2] David A Mix Barrington, Richard Beigel, and Steven Rudich. 1994. Representing Boolean functions as polynomials modulo composite numbers. Computational Complexity 4, 4 (1994), 367-382.

[3] Libor Barto, Andrei A. Krokhin, and Ross Willard. 2017. Polymorphisms, and How to Use Them. In The Constraint Satisfaction Problem: Complexity and Approximability, Andrei A. Krokhin and Stanislav Zivny (Eds.). Dagstuhl Follow-Ups, Vol. 7. Schloss Dagstuhl - Leibniz-Zentrum fuer Informatik, 1-44. https://doi.org/10.4230/DFU.Vol7.15301.1

[4] Abhishek Bhowmick, Zeev Dvir, and Shachar Lovett. 2014. New bounds for matching vector families. SIAM F. Comput. 43, 5 (2014), 1654-1683.

[5] A. Bulatov, P. Jeavons, and A. Krokhin. 2005. Classifying the Complexity of Constraints Using Finite Algebras. SIAM 7. Comput. 34, 3 (Jan. 2005), 720-742. https://doi.org/10.1137/S0097539700376676

[6] Andrei A. Bulatov. 2006. A dichotomy theorem for constraint satisfaction problems on a 3-element set. f. ACM 53, 1 (2006), 66-120. https://doi.org/10.1145/ 1120582.1120584

[7] Andrei A. Bulatov. 2017. A Dichotomy Theorem for Nonuniform CSPs, See [29], 319-330. https://doi.org/10.1109/FOCS.2017.37

[8] Andrei A. Bulatov and Daniel Marx. 2010. The complexity of global cardinality constraints. Logical Methods in Computer Scienced Volume 6, Issue 4 (Oct. 2010) https://doi.org/10.2168/LMCS-6(4:4)2010

[9] Arkadev Chattopadhyay, Navin Goyal, Pavel Pudlák, and Denis Thérien. 2006 Lower bounds for circuits with MOD m gates. In 47th Annual IEEE Symposium on Foundations of Computer Science (FOCS 2006), 21-24 October 2006, Berkeley, California, USA, Proceedings. 709-718. https://doi.org/10.1109/FOCS.2006.46

[10] Arkadev Chattopadhyay and Avi Wigderson. 2009. Linear Systems over Composite Moduli. In 50th Annual IEEE Symposium on Foundations of Computer Science, FOCS 2009, October 25-27, 2009, Atlanta, Georgia USA. 43-52. https //doi.org/10.1109/FOCS.2009.17

[11] Gil Cohen and Avishay Tal. 2015. Two Structural Results for Low Degree Polynomials and Applications. Approximation, Randomization, and Combinatorial Optimization. Algorithms and Techniques (2015), 680

[12] Nadia Creignou, Henning Schnoor, and Ilka Schnoor. 2010. Nonuniform Boolean Constraint Satisfaction Problems with Cardinality Constraint. ACM Trans. Comput. Logic 11, 4, Article 24 (July 2010), 32 pages. https://doi.org/10.1145/1805950. 1805954

[13] Zeev Dvir, Parikshit Gopalan, and Sergey Yekhanin. 2011. Matching vector codes. SIAM f. Comput. 40, 4 (2011), 1154-1178.

[14] Zeev Dvir and Sivakanth Gopi. 2016. 2-Server PIR with subpolynomial communication. Fournal of the ACM (FACM) 63, 4 (2016), 39.
[15] Klim Efremenko. 2012. 3-query locally decodable codes of subexponential length. SIAM F. Comput. 41, 6 (2012), 1694-1703.

[16] Tomás Feder and Moshe Y. Vardi. 1998. The Computational Structure of Monotone Monadic SNP and Constraint Satisfaction: A Study through Datalog and Group Theory. SIAM F. Comput. 28, 1 (1998), 57-104. https://doi.org/10.1137/ S0097539794266766

[17] Parikshit Gopalan. 2009. A note on Efremenko's Locally Decodable Codes.. In Electronic Colloquium on Computational Complexity (ECCC), Vol. 16.

[18] Parikshit Gopalan. 2014. Constructing Ramsey graphs from Boolean function representations. Combinatorica 34, 2 (2014), 173-206.

[19] Vince Grolmusz. 2000. Superpolynomial size set-systems with restricted intersections mod 6 and explicit Ramsey graphs. Combinatorica 20, 1 (2000), 71-86.

[20] Venkatesan Guruswami and Euiwoong Lee. 2016. Complexity of Approximating CSP with Balance / Hard Constraints. Theory of Computing Systems 59, 1 (01 Jul 2016), 76-98. https://doi.org/10.1007/s00224-015-9638-0

[21] Jonathan Katz and Luca Trevisan. 2000. On the efficiency of local decoding procedures for error-correcting codes. In Proceedings of the thirty-second annual ACM symposium on Theory of computing. ACM, 80-86.

[22] Iordanis Kerenidis and Ronald De Wolf. 2004. Exponential lower bound for 2-query locally decodable codes via a quantum argument. 7. Comput. System Sci. 69, 3 (2004), 395-420.

[23] Tianren Liu and Vinod Vaikuntanathan. 2018. Breaking the circuit-size barrier in secret sharing. In Proceedings of the 50th Annual ACM SIGACT Symposium on Theory of Computing, STOC 2018, Los Angeles, CA, USA, fune 25-29, 2018. 699-708. https://doi.org/10.1145/3188745.3188936

[24] Dana Moshkovitz and Ran Raz. 2010. Two-query PCP with subconstant error. fournal of the ACM (FACM) 57, 5 (2010), 29.

[25] Martin Nägele, Benny Sudakov, and Rico Zenklusen. 2018. Submodular minimization under congruency constraints. In Proceedings of the Twenty-Ninth Annual ACM-SIAM Symposium on Discrete Algorithms. Society for Industrial and Applied Mathematics, 849-866.

[26] Emil L Post. 1941. The two-valued iterative systems of mathematical logic. Number 5 in Annals of Mathematics Studies. Princeton University Press.

[27] Thomas J. Schaefer. 1978. The Complexity of Satisfiability Problems. In Proceedings of the Tenth Annual ACM Symposium on Theory of Computing (STOC '78). ACM, New York, NY, USA, 216-226. https://doi.org/10.1145/800133.804350

[28] Gábor Tardos and DA Mix Barrington. 1998. A lower bound on the MOD 6 degree of the OR function. Computational Complexity 7, 2 (1998), 99-108.

[29] Chris Umans (Ed.). 2017. 58th IEEE Annual Symposium on Foundations of Computer Science, FOCS 2017, Berkeley, CA, USA, October 15-17, 2017. IEEE Computer Society. http://ieeexplore.ieee.org/xpl/mostRecentIssue.jsp?punumber=8100284

[30] Sergey Yekhanin. 2008. Towards 3-query locally decodable codes of subexponential length. Fournal of the ACM ( $7 A C M) 55,1$ (2008), 1.

[31] Dmitriy Zhuk. 2017. A Proof of CSP Dichotomy Conjecture, See [29], 331-342. https://doi.org/10.1109/FOCS.2017.38 